\title{
Impact of tides on the transit-timing fits to the TRAPPIST-1 system
}

\author{
E. Bolmont ${ }^{1}$, B.-O. Demory ${ }^{2}$, S. Blanco-Cuaresma ${ }^{3}$, E. Agol ${ }^{4}$, S. L. Grimm ${ }^{2}$, P. Auclair-Desrotour ${ }^{2}$, \\ F. Selsis ${ }^{5}$, and A. Leleu ${ }^{2}$
}

\author{
${ }^{1}$ Observatoire de Genève, Université de Genève, 51 chemin des Maillettes, C1290 Sauverny, Switzerland \\ e-mail: emeline.bolmont@unige.ch \\ 2 Physikalisches Institut, Universität Bern, Gesellschaftsstrasse 6, 3012 Bern, Switzerland \\ ${ }^{3}$ Harvard-Smithsonian Center for Astrophysics, 60 Garden Street, Cambridge, MA 02138, USA \\ ${ }^{4}$ Astronomy Department, University of Washington, Seattle, WA 98195, USA \\ ${ }^{5}$ Laboratoire d'astrophysique de Bordeaux, Université de Bordeaux, CNRS, B18N, allée Geoffroy Saint-Hilaire, 33615 Pessac, \\ France
}

Received 21 January 2020 / Accepted 5 February 2020

\begin{abstract}
Transit timing variations (TTVs) can be a very efficient way of constraining masses and eccentricities of multi-planet systems. Recent measurements of the TTVs of TRAPPIST-1 have led to an estimate of the masses of the planets, enabling an estimate of their densities and their water content. A recent TTV analysis using data obtained in the past two years yields a 34 and $13 \%$ increase in mass for TRAPPIST-1b and c, respectively. In most studies to date, a Newtonian $N$-body model is used to fit the masses of the planets, while sometimes general relativity is accounted for. Using the Posidonius $N$-body code, in this paper we show that in the case of the TRAPPIST-1 system, non-Newtonian effects might also be relevant to correctly model the dynamics of the system and the resulting TTVs. In particular, using standard values of the tidal Love number $k_{2}$ (accounting for the tidal deformation) and the fluid Love number $k_{2 f}$ (accounting for the rotational flattening) leads to differences in the TTVs of TRAPPIST-1b and c that are similar to the differences caused by general relativity. We also show that relaxing the values of tidal Love number $k_{2}$ and the fluid Love number $k_{2 f}$ can lead to TTVs which differ by as much as a few $10 \mathrm{~s}$ on a 3-4-yr timescale, which is a potentially observable level. The high values of the Love numbers needed to reach observable levels for the TTVs could be achieved for planets with a liquid ocean, which if detected might then be interpreted as a sign that TRAPPIST-1b and TRAPPIST-1c could have a liquid magma ocean. For TRAPPIST-1 and similar systems the models to fit the TTVs should potentially account for general relativity, for the tidal deformation of the planets, for the rotational deformation of the planets, and to a lesser extent for the rotational deformation of the star, which would add up to $7 \times 2+1=15$ additional free parameters in the case of TRAPPIST- 1
\end{abstract}

Key words. planets and satellites: dynamical evolution and stability - planets and satellites: individual: TRAPPIST-1 planet-star interactions

\section{Introduction}

The measurement of transit timing variations (TTVs) in the context of multi-transiting planet systems can be a very efficient method for deriving the dynamical parameters of a planetary system, such as mass and eccentricity (see Agol \& Fabrycky 2018, for a review). The TRAPPIST-1 system has been intensely monitored by TRAPPIST, K2, and Spitzer, which led to estimates of the masses of the planets by Grimm et al. (2018). Recently additional Spitzer observations were obtained thanks to the Spitzer proposal no. 14223 (Agol et al. 2019).

In most studies on TTVs the model used is an $N$-body model assuming point-mass and Newtonian dynamics, sometimes with a prescription for general relativity (as in Grimm et al. 2018; Jordán \& Bakos 2008; Pál \& Kocsis 2008). Theoretical studies have considered the possible impact of tides and quadrupole distortion on transit times (Miralda-Escudé 2002; Heyl \& Gladman 2007). However, the influence of tides has never been consistently taken into account in a multi-planet context.

Some studies do take into account tidal decay (e.g., Maciejewski et al. 2018), but decay typically occurs on timescales that are much longer than the typical duration of the observations available for TRAPPIST-1. However, tidal forces are not only a dissipative effect (which drives migration and spin evolution), there is also a non-dissipative effect which depends on the real part of the complex Love number of degree- $2, k_{2}$, which quantifies the shape of the tidal deformation. This deformation can lead to a precession of the orbit, which can lead to TTVs. In addition, for fast rotating planets, rotational flattening can also drive a precession of the orbit, which in turn can lead to TTVs. These effects have been considered in systems with a single hot-Jupiter planet (see Ragozzine \& Wolf 2009 , for a comparative study of each effect). The precession of the orbit leads to observable TTVs, which then can inform the internal structure of the planet through the determination of the Love number.

However, these effects are usually never taken into account when investigating the TTVs of multi-planetary systems. We show that in the context of TRAPPIST-1 (Gillon et al. 2017, 2016), the inclusion of tidal forces may lead to an observable TTV signal. In contrast with TRAPPIST- $1 \mathrm{~d}-\mathrm{h}$, planets $\mathrm{b}$ and $\mathrm{c}$ are in proximity to a higher-order resonance (increasing the frequency of the TTV pattern modulation; see Agol et al. 2005) and exhibit small TTV amplitudes (2-5 min); both effects inflate the uncertainties on the masses and eccentricities, as shown in Grimm et al. (2018). Interestingly, a recent TTV analysis using 
data obtained in the past two years yields a $34 \%$ increase in mass for TRAPPIST-1b and a $13 \%$ increase in mass for TRAPPIST-1c (in prep.) compared to Grimm et al. (2018). These mass increases of the two inner planets drew our attention to physical processes that could impact the planet physical and orbital parameters on secular timescales. As the parameters for the other planets have remained relatively insensitive to the addition of new data, two hypotheses remain. A first possibility is that the changing masses are due to an incomplete sampling of the TTV pattern that should resolve as new data are included. A second possibility is that dynamical models are missing physical processes that impact the close-in planets more strongly, such as tides and rotational flattening.

We show in this paper that the precession caused by general relativity, by tidal deformation, and by rotational flattening could lead to significantly different TTVs for the two inner planets of TRAPPIST-1.

\section{Simulation setup}

We use Posidonius ${ }^{1}$ v2019.07.30 (Blanco-Cuaresma \& Bolmont 2017; Blanco-Cuaresma \& Bolmont, in prep.), an $N$-body code which allows users to take into account additional forces and torques: tidal forces and torques, rotational flattening forces and torques, and general relativity (Bolmont et al. 2015). As in Bolmont et al. (2015), tides are computed between a planet and the star independently of the other planets, and the planet-planet tides are not taken into account (which is justified, see Hay \& Matsuyama 2019). In Posidonius, we use the integrator IAS15 (Rein \& Spiegel 2015) to compute the evolution of the system for 1500 days, which is approximately the time range available from all the observations collected from the system, and we fix the maximum time step allowed at 0.01 day $=14 \mathrm{~min}$. We tested the convergence of our code with time steps of 0.005 day and 0.001 day, and find that the transit timings are stable to a precision of better than $10^{-6} \mathrm{~s}$.

\subsection{Tidal model}

Posidonius accounts for equilibrium tides following the prescription of Bolmont et al. (2015), which is an implementation of the constant time-lag model (Mignard 1979; Hut 1981; Eggleton et al. 1998). The equilibrium tide is the result of the hydrostatic adjustment of a body; instead, the dynamical tide is the tidal response corresponding to the propagation of waves, for example inertial waves in the convective region of stars (see Zahn 1975) or gravito-inertial waves in a planetary liquid layer (see Auclair-Desrotour et al. 2019).

We review here the expressions for the tidal force and torques. Let us consider a star, defined by its mass $M_{\star}$, its radius $R_{\star}$, its degree- 2 potential Love number $k_{2, \star}$, its (constant) time lag $\Delta \tau_{\star}$, and its spin vector $\boldsymbol{\Omega}_{\star}$. Let us consider one planet, $\mathrm{j}$, orbiting the star at a distance $r_{\mathrm{j}}$. The planet is defined by its mass $M_{\mathrm{p}_{\mathrm{j}}}$, its radius $R_{\mathrm{p}_{\mathrm{j}}}$, its degree-2 potential Love number $k_{2, \mathrm{p}_{\mathrm{j}}}$, its (constant) time lag $\Delta \tau_{\mathrm{p}_{\mathrm{j}}}$, and its spin vector $\boldsymbol{\Omega}_{\mathrm{p}_{\mathrm{j}}}$.

Let us define $\mathbf{F}_{\text {diss, } p_{\mathrm{j}}}$ and $\mathbf{F}_{\text {nodiss, }}$ as the dissipative part and the non-dissipative part, respectively, of the force exerted on planet $\mathrm{j}$ due to the planetary tide as (Bolmont et al. 2015)

$\mathbf{F}_{\text {nodiss, } \mathrm{p}_{\mathrm{j}}}=\frac{-3 \mathcal{G}}{r_{\mathrm{j}}^{7}} M_{\star}^{2} k_{2, \mathrm{p}_{\mathrm{j}}} R_{\mathrm{p}_{\mathrm{j}}}^{5} \boldsymbol{e}_{\boldsymbol{r}_{\mathrm{j}}}$,

1 https://www . blancocuaresma.com/s/posidonius

$$
\begin{aligned}
\mathbf{F}_{\text {diss, } \mathrm{p}_{\mathrm{j}}}= & -9 \mathcal{G} \frac{\dot{r}_{\mathrm{j}}}{r_{\mathrm{j}}^{8}} M_{\star}^{2} R_{\mathrm{p}_{\mathrm{j}}}^{5} k_{2, \mathrm{p}_{\mathrm{j}}} \Delta \tau_{\mathrm{p}_{\mathrm{j}}} \boldsymbol{e}_{r_{\mathrm{j}}} \\
& +3 \mathcal{G} \frac{M_{\star}^{2} R_{\mathrm{p}_{\mathrm{j}}}^{5}}{r_{\mathrm{j}}^{7}} k_{2, \mathrm{p}_{\mathrm{j}}} \Delta \tau_{\mathrm{p}_{\mathrm{j}}}\left(\mathbf{\Omega}_{\mathrm{p}_{\mathrm{j}}}-\dot{\boldsymbol{\theta}}_{\mathrm{j}}\right) \times \boldsymbol{e}_{r_{\mathrm{j}}},
\end{aligned}
$$

where $\boldsymbol{e}_{\boldsymbol{r}_{\mathrm{j}}}$ is the unit vector $\boldsymbol{r}_{\mathrm{j}} / r_{\mathrm{j}}$ and $\dot{\boldsymbol{\theta}}_{\mathrm{j}}$ is a vector collinear with the orbital angular momentum of planet $j$, the norm of which is equal to the time derivative of the true anomaly. Let us define $\mathbf{F}_{\text {diss }, \star}$ and $\mathbf{F}_{\text {nodiss, } \star}$ as the dissipative part and the non-dissipative part, respectively, of the force exerted on planet $j$ due to the stellar tide as

$$
\begin{aligned}
\mathbf{F}_{\text {nodiss }, \star} & =\frac{-3 \mathcal{G}}{r_{\mathrm{j}}^{7}} M_{\mathrm{p}_{\mathrm{j}}}^{2} k_{2, \star} R_{\star}^{5} \boldsymbol{e}_{r_{\mathrm{j}}}, \\
\mathbf{F}_{\mathrm{diss}, \star}= & -9 \mathcal{G} \frac{\dot{r}_{\mathrm{j}}}{r_{\mathrm{j}}^{8}} M_{\mathrm{p}_{\mathrm{j}}}^{2} R_{\star}^{5} k_{2, \star} \Delta \tau_{\star} \boldsymbol{e}_{r_{\mathrm{j}}} \\
& +3 \mathcal{G} \frac{M_{\mathrm{p}_{\mathrm{j}}}^{2} R_{\star}^{5}}{r_{\mathrm{j}}^{7}} k_{2, \star} \Delta \tau_{\star}\left(\mathbf{\Omega}_{\star}-\dot{\boldsymbol{\theta}}_{\mathrm{j}}\right) \times \boldsymbol{e}_{r_{\mathrm{j}}} .
\end{aligned}
$$

The total force as a result of the tides acting on a planet $\mathrm{j}$ is therefore given by the sum of these contributions (Bolmont et al. 2015)

$\mathbf{F}_{\mathrm{p}_{\mathrm{j}}}^{\mathbf{T}}=\mathbf{F}_{\text {diss, } \mathrm{p}_{\mathrm{j}}}+\mathbf{F}_{\text {nodiss }, \mathrm{p}_{\mathrm{j}}}+\mathbf{F}_{\text {diss }, \star}+\mathbf{F}_{\text {nodiss, },}$.

\subsection{Rotational flattening model}

To account for rotational flattening, we also follow here the prescription of Bolmont et al. (2015), which assumes that the deformation due to the rotational flattening results in a triaxial ellipsoid symmetric with respect to the rotation axis (Murray \& Dermott 1999). This deformation is quantified by a parameter, $J_{2}$, which depends on the radius, mass, and spin of the body and on the potential Love number of degree- 2 for a perfectly fluid body (which we call here the fluid Love number; Correia \& Rodríguez 2013). We define this parameter for planet $\mathrm{j}$ and the star as

$J_{2, \mathrm{p}_{\mathrm{j}}}=k_{2 f, \mathrm{p}_{\mathrm{j}}} \frac{\Omega_{\mathrm{p}_{\mathrm{j}}}^{2} R_{\mathrm{p}_{\mathrm{j}}}^{3}}{3 \mathcal{G} M_{\mathrm{p}_{\mathrm{j}}}}$,
$J_{2, \star}=k_{2 f, \star} \frac{\Omega_{\star}^{2} R_{\star}^{3}}{3 \mathcal{G} M_{\star}}$.

Let us define $\mathbf{F}_{\text {rot, } \mathrm{p}_{\mathrm{j}}}$ the force exerted on planet $\mathrm{j}$ due to the rotational flattening of planet $j$ and $\mathbf{F}_{\text {rot, } \star}$ the force exerted on planet $j$ due to the rotational flattening of the star as (Murray \& Dermott 1999; Correia et al. 2011)

$\mathbf{F}_{\text {rot, } \mathrm{p}_{\mathrm{j}}}=\left(-\frac{3}{r_{\mathrm{j}}^{5}} C_{\mathrm{p}_{\mathrm{j}}}+\frac{15}{r_{\mathrm{j}}^{7}} C_{\mathrm{p}_{\mathrm{j}}} \frac{\left(\boldsymbol{r}_{\mathrm{j}} \cdot \boldsymbol{\Omega}_{\mathrm{p}_{\mathrm{j}}}\right)^{2}}{\Omega_{\mathrm{p}_{\mathrm{j}}}^{2}}\right) \boldsymbol{r}_{\mathrm{j}}-\frac{6}{r_{\mathrm{j}}^{5}} C_{\mathrm{p}_{\mathrm{j}}} \frac{\boldsymbol{r}_{\mathrm{j}} \cdot \boldsymbol{\Omega}_{\mathrm{p}_{\mathrm{j}}}}{\Omega_{\mathrm{p}_{\mathrm{j}}}^{2}} \mathbf{\Omega}_{\mathrm{p}_{\mathrm{j}}}$,
$\mathbf{F}_{\mathrm{rot}, \star}=\left(-\frac{3}{r_{\mathrm{j}}^{5}} C_{\star}+\frac{15}{r_{\mathrm{j}}^{7}} C_{\star} \frac{\left(\boldsymbol{r}_{\mathrm{j}} \cdot \mathbf{\Omega}_{\star}\right)^{2}}{\Omega_{\star}^{2}}\right) \boldsymbol{r}_{\mathrm{j}}-\frac{6}{r_{\mathrm{j}}^{5}} C_{\star} \frac{\boldsymbol{r}_{\mathrm{j}} \cdot \boldsymbol{\Omega}_{\star}}{\Omega_{\star}^{2}} \boldsymbol{\Omega}_{\star}$,

where $C_{\star}$ and $C_{\mathrm{p}_{\mathrm{j}}}$ are defined as follows:

$C_{\mathrm{p}_{\mathrm{j}}}=\frac{1}{2} \mathcal{G} M_{\mathrm{p}_{\mathrm{j}}} M_{\star} J_{2, \mathrm{p}_{\mathrm{j}}} R_{\mathrm{p}_{\mathrm{j}}}^{2}$,

$C_{\star}=\frac{1}{2} G M_{\mathrm{p}_{\mathrm{j}}} M_{\star} J_{2, \star} R_{\star}^{2}$. 
The resulting force on planet $\mathrm{j}$ due to the rotational deformation of both the star and planet $j$ is the sum of the two contributions:

$\mathbf{F}_{\mathrm{p}_{\mathrm{j}}}^{\mathbf{R}}=\mathbf{F}_{\text {rot, } \mathrm{p}_{\mathrm{j}}}+\mathbf{F}_{\mathrm{rot}, \star} \cdot$

\subsection{General relativity}

We use three different prescriptions for general relativity: Kidder (1995), which is the one used in Mercury-T (Bolmont et al. 2015; Anderson et al. 1975; and Newhall et al. 1983).

The prescription of Kidder (1995) was designed for two bodies, and Posidonius takes into account the post-Newtonian, the spin-orbit, and the post ${ }^{2}$-Newtonian contributions to the total acceleration (Eqs. (2.2b)-(2.2d), respectively), as well as the spin precession equations for both bodies (Eqs. (2.4a) and $(2.4 \mathrm{~b}))$.

The prescription of Anderson et al. (1975) accounts for the post-Newtonian acceleration of two bodies. We refer the reader to Eq. (12) in Anderson et al. (1975) where the expression of this acceleration is given.

The prescription of Newhall et al. (1983) is more complete insofar as it accounts for the post-Newtonian effect between all bodies. We refer to Eq. (1) in Newhall et al. (1983), which gives the point-mass acceleration. Posidonius accounts for this acceleration, except for the last term which accounts for the perturbation of five solar system asteroids.

\section{Transit timing variations}

We performed simulations of the TRAPPIST-1 system switching on and off these various effects: the effect of the planetary tide (by varying the Love number $k_{2, \mathrm{p}}$ and the time lag $\tau_{\mathrm{p}}$ ), the effect of the stellar tide (by varying the Love number $k_{2, \star}$ ), the effect of the rotational flattening of the planets (by varying the fluid Love number $k_{2 f, p}$ ), the effect of the rotational flattening of the star (by varying the fluid Love number $k_{2 f, \star}$ ), and the effect of general relativity. We list in Table 1 the reference values of the parameters varied here, and we refer the reader to Appendix A for the parameters that remain constant in our simulations, including the initial orbital elements for the planets. We tested the three different prescriptions of the general relativity introduced in Sect. 2.3. They gave very similar results so that in the following we compare the other effects with respect to the simulations performed using the prescription of Kidder (1995).

The planetary reference values were taken to be representative of the Earth; in particular, the quantity $k_{2, \mathrm{p}} \Delta \tau_{\mathrm{p}}$ is equal to 213 s (Neron de Surgy \& Laskar 1997). The stellar reference values were chosen to be representative of fully convective M dwarfs (Bolmont et al. 2015).

For all the simulations we performed, we calculated the transit timing variations (Agol \& Fabrycky 2018) as follows: (i) for each transit we find the time of the transit mid-time by performing an interpolation to find the precise time a given planet crosses a reference direction. This corresponds to the "Observed transit time" $O$; (ii) we evaluate the "Calculated transit times" $C$ by performing a linear $\mathrm{fit}^{2}$ of the transit times calculated in step i) over the total number of transits; (iii) we calculate the difference $O-C$ to obtain the TTVs as a function of the epoch (or transit number).

To quantify the impact of each additional effect on the simulated TTVs, we compute the difference between the TTVs

2 We use the function LinearRegression from the linear_model package of scikit-learn.
Table 1. Reference values for the parameters we vary in this study.

\begin{tabular}{cc}
\hline \hline Parameter & Value \\
\hline$k_{2 f, \mathrm{p}}^{\text {ref }}$ & 0.9532 \\
$k_{2, \mathrm{p}}^{\text {ref }}$ & 0.299 \\
$\Delta \tau_{\mathrm{p}}^{\text {ref }}(\mathrm{s})$ & 712.37 \\
\hline$k_{2 f, \star}^{\text {ref }}$ & 0.307 \\
$k_{2, \star}^{\text {ref }}$ & 0.307 \\
\hline
\end{tabular}

Notes. We assume that all planets in the system have the same potential Love number of degree- $2 k_{2 f, \mathrm{p}}$, fluid Love number $k_{2, \mathrm{p}}$, and time lag $\Delta \tau_{\mathrm{p}}$.

calculated taking into account an additional effect and the TTVs obtained for a Newtonian $N$-body integration.

\section{Influence of each effect on the TTVs}

We performed a set of six simulations of the TRAPPIST-1 system to test the impact of the additional effects listed in Sect. 2, and we compare each with a Newtonian $N$-body simulation. One after another, we explored the effect of each parameter using the reference values in Table 1 and general relativity. We tested the influence of the dissipative part of the planetary tide by assuming $\Delta \tau_{\mathrm{p}}=1 \times \Delta \tau_{\mathrm{p}}^{\text {ref }}$ for all planets, with all the other parameters set to zero. We repeated the operation for the non-dissipative part of the planetary tide (through $k_{2, \mathrm{p}}$, equal for all planets), the rotational flattening of the planets (through $k_{2 f, \mathrm{p}}$, equal for all planets), the non-dissipative part of the stellar tide (through $k_{2, \star}$ ), the rotational flattening of the star (through $k_{2 f, \star}$ ), and for general relativity.

Figure 1 shows the results for planets $b$ to $d$. The top panels of Fig. 1 show the transit timing variations for the three planets for the seven simulations, and the bottom panels show the difference between the TTVs and the TTVs corresponding to the pure $N$-body simulation. The different additional effects have a very limited impact on the shape of the TTVs, but computing the difference with the pure $N$-body case reveals the amplitude of each effect.

For TRAPPIST-1b (T-1b), the dominant effects are the nondissipative part of the planetary tide (green in Fig. 1a) and general relativity (pink), respectively accounting for a difference in TTVs of about $-0.63 \mathrm{~s}$ and $0.56 \mathrm{~s}$ after 1500 days. The effect of the rotational flattening of the planets (red) plays a smaller role, but still accounts for more than half the amplitude due to the non-dissipative part of the planetary tide with a difference of $-0.33 \mathrm{~s}$. The effect of the dissipative part of the planetary tide (orange) and the non-dissipative part of the stellar tide (purple) are completely negligible (accounting for a difference $\sim 1 \times 10^{-3}$ and $\sim 1 \times 10^{-4}$ s, respectively), which is in agreement with Ragozzine \& Wolf (2009). The effect of the rotational flattening of the star (brown) is much smaller (accounting for a difference of $-0.057 \mathrm{~s}$ ), but might contribute to a lesser extent. Accounting for all effects (gray) leads to an absolute difference of $-0.45 \mathrm{~s}$ at the end of the 1500-day simulation. The effects of general relativity and the non-dissipative part of the planetary tide almost cancel each other out, while the amplitude is determined by the effect of the rotational flattening of the planet (red curve) and of the star (brown curve).

For TRAPPIST-1c (T-1c), the dominant effects are the nondissipative part of the planetary tide (accounting for a difference of $1 \mathrm{~s}$, in green in Fig.1b), followed by the effect of the 
(a) TRAPPIST-1b
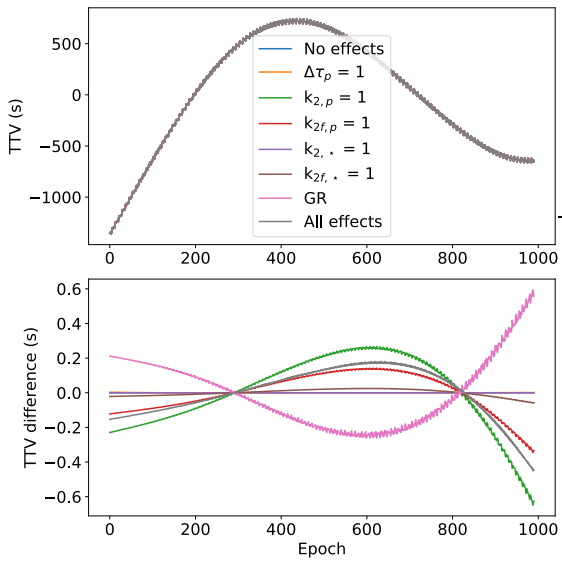

(b) TRAPPIST-1c
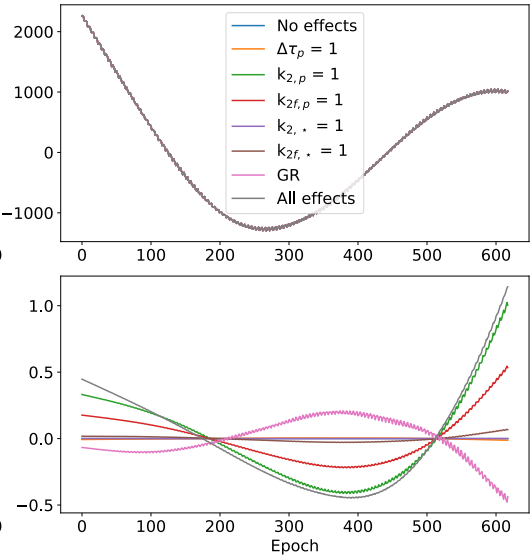

(c) TRAPPIST-1d
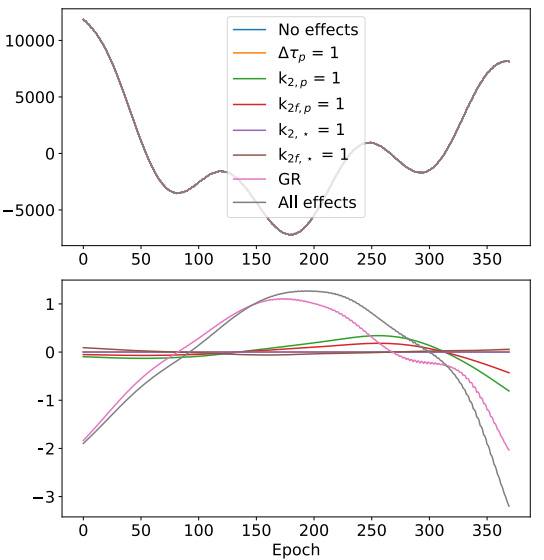

Fig. 1. Impact of various additional effects on the TTVs of $(a)$ planet $b,(b)$ planet $\mathrm{c}$, and $(c)$ planet d. Top panel: transit timing variations for a pure $N$-body simulation (blue); for a simulation using $\Delta \tau_{\mathrm{p}}=1 \times \Delta \tau_{\mathrm{p}}^{\text {ref }}$ for all planets (orange), using $k_{2, \mathrm{p}}=1 \times k_{2, \mathrm{p}}^{\text {ref }}$ for all planets (green), using $k_{2 f, \mathrm{p}}=$ $1 \times k_{2 f, \mathrm{p}}^{\text {ref }}$ for all planets (red), using $k_{2, \star}=1 \times k_{2, \star}^{\text {ref }}$ (purple), using $k_{2 f, \star}=1 \times k_{2 f, \star}^{\text {ref }}$ (brown), and where we only consider the general relativity (pink); and for a simulation where all effects are taken into account. Bottom panel: corresponding TTVs differences with the Newtonian $N$-body case.

rotational flattening of the planet $(0.53 \mathrm{~s}$, in red), followed by the effect of general relativity $(-0.44 \mathrm{~s}$, pink). The effect of the rotational flattening of the star accounts for $0.067 \mathrm{~s}$ (purple) and the effect of the dissipative part of the planetary tide remains negligible $(0.013 \mathrm{~s}$, orange). As with $\mathrm{T}-1 \mathrm{~b}$, to reproduce the difference observed when all effects are taken into account (in gray in Fig.1b), we need to account for the non-dissipative part of the planetary tide, the rotational flattening of the planet, general relativity, and the rotational flattening of the star to a lesser extent. We note that the precession of the orbits due to the rotational flattening depends on the square of the spin frequency of the considered body (Ragozzine \& Wolf 2009). Here we use a rotation period of 3.3 days for TRAPPIST-1 (Luger et al. 2017). It is possible that the rotation is slower (as the period distribution of nearby late M dwarfs shows, Newton et al. 2016), in which case the contribution of the rotational flattening of the star would be even less important.

For TRAPPIST-1d (T-1d), Fig. 1c shows that the dominant effect is general relativity $(-2.03 \mathrm{~s}$, in pink). The effect of the non-dissipative part of the planetary tide (accounting for $-0.81 \mathrm{~s}$, green) and the effect of the rotational flattening of the planet (accounting for $-0.43 \mathrm{~s}$, red) should probably also be taken into account. This is also true for all the external planets: the amplitude due to general relativity is much higher, but at the same time not accounting for at least the non-dissipative part of the planetary tide and the rotational flattening of the planet leads to small offsets (see Appendix B, Fig B.1).

\section{Potential observable effects}

We performed simulations for which we varied the potential Love number $k_{2, \mathrm{p}}$ and the fluid Love number $k_{2 f, \mathrm{p}}$ over a wide range. We first treat these parameters as free parameters with no limitations on their value and then we discuss the validity of this approach in Sect. 6.

As in Sect. 4, we always assume the same value of the Love numbers for all planets. We vary the parameter $k_{2, \mathrm{p}}$ from $1 \times k_{2, \mathrm{p}}^{\mathrm{ref}}=0.299$ to $50 \times k_{2, \mathrm{p}}^{\mathrm{ref}}=14.95$; the impact on the TTV differences with the pure $N$-body case can be seen in the top panel of Fig. 2a for T-1b and in the top panel of Fig. 2b for T-1c.

Considering the highest value of the tidal Love number leads to a difference in TTVs after 1500 days of $-31.7 \mathrm{~s}$ for T-1b and $50.7 \mathrm{~s}$ for $\mathrm{T}-1 \mathrm{c}$. The amplitude of these effects are then comparable to the precision achievable today on the observed TTVs of the two inner planets. If the tidal Love number could reach such high values, the effect of the non-dissipative part of the planetary tide (the tidal deformation of T-1b and T-1c) would therefore be detectable.

Similarly, we vary the parameter $k_{2 f, \mathrm{p}}$ from $1 \times k_{2 f, \mathrm{p}}^{\text {ref }}=0.9532$ to $50 \times k_{2 f, \mathrm{p}}^{\mathrm{ref}}=47.66$, and the impact on the TTV differences with the pure $N$-body case can be seen in the bottom panel of Fig. 2 a for T-1b and in the bottom panel of Fig. 2b for T-1c. Assuming the highest value of the fluid Love number leads to a difference in TTVs after 1500 days of $-17.3 \mathrm{~s}$ for T-1b and $27.7 \mathrm{~s}$ for T-1c.

Considering all the important effects given in Sect. 4 and relaxing the range of possible Love numbers might therefore be a way to settle the question of the increasing masses of $\mathrm{T}-1 \mathrm{~b}$ and $\mathrm{T}-1 \mathrm{c}$ and to settle the two hypotheses given in the introduction: is it a sampling problem or are we missing dynamical processes? The answer to this question depends upon the potential degeneracy of these effects with varying the $N$-body parameters, and on the duration and precision of the transit timing measurements. We do not explore these effects here.

\section{Discussion}

We showed that for systems like TRAPPIST-1 the effect of the tidal deformation of the planets (through the planetary tidal Love number $k_{2, \mathrm{p}}$ ), the effect of the rotational deformation of the planets (through the planetary fluid Love number $k_{2 f, \mathrm{p}}$ ), and the effect of the rotational flattening of the star (through the stellar fluid Love number $k_{2 f, \star}$ ) can impact the TTVs of the two inner planets at the same order of magnitude as the general relativity, if we assume "standard" values for these parameters. We also showed that the tidal dissipation (responsible for the misalignment which drives long term tidal evolution) does not significantly impact the TTVs of the system over the short observation time that we simulated. By relaxing the assumptions on the planetary tidal and fluid Love numbers, we also showed that a high Love number can lead to differences in TTV on the order of $\sim 10 \mathrm{~s}$. This difference is potentially observable with the current precision we have on the transit timings, unless there is significant degeneracy with other $N$-body parameters.

However, it is commonly accepted that a tidal Love number cannot exceed 1.5, which corresponds to a homogeneous 
(a) TRAPPIST-1b

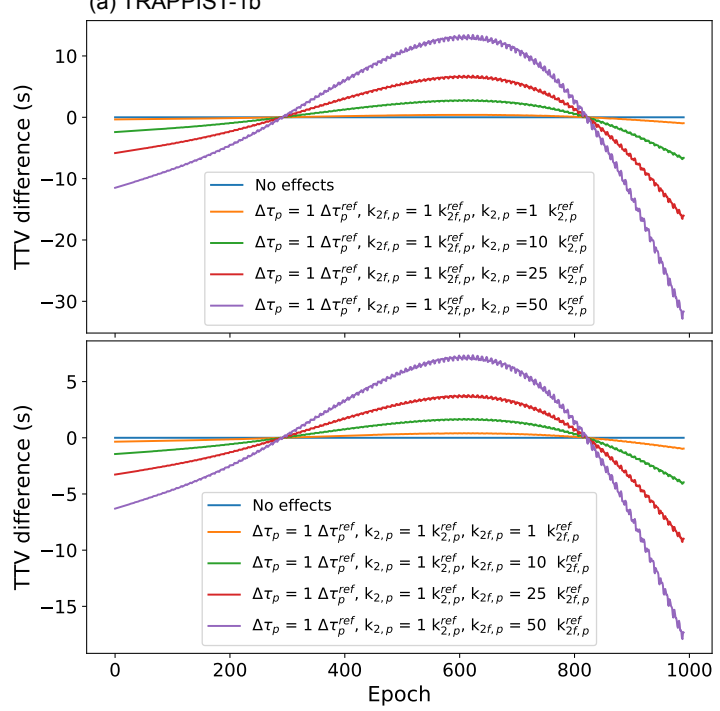

(b) TRAPPIST-1c

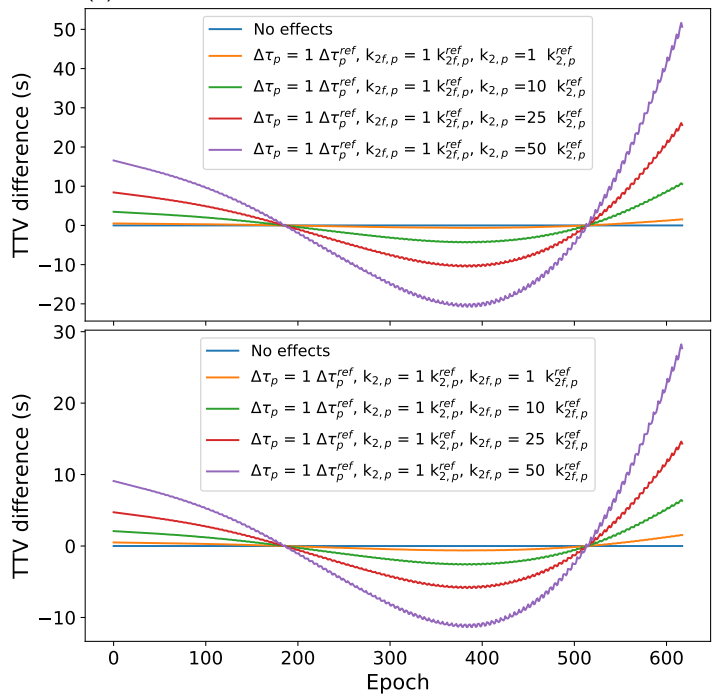

Fig. 2. Differences in TTVs with the pure $N$-body case for $(a)$ planet b and $(b)$ planet c. Top panel: fixing the planetary fluid Love number and the dissipation to their reference values (see Table 1), the potential Love number $k_{2, \mathrm{p}}$ is varied between 1 and $50 \times k_{2, \mathrm{p}}^{\text {ref }} \sim 15$. Bottom panel: fixing the planetary Love number and the dissipation to their reference values, the potential Love number $k_{2 f, \mathrm{p}}$ is varied between 1 and $50 \times k_{2 f, \mathrm{p}}^{\text {ref }} \sim 48$.

body. This means that the physical range of our study should encompass maximum values, which are $5 \times k_{2, \mathrm{p}}^{\text {ref }}=5 \times 0.299$. Limiting ourselves to this value would entail a difference in TTVs for T-1b of less than $2.5 \mathrm{~s}$, which is below the precision we can achieve today. On the other hand, it is known that if a planet has a liquid layer (liquid water ocean, or liquid magma ocean), the response of the body becomes more complex; in particular, it becomes highly dependent on the excitation frequency. Specifically, if a frequency excites a resonant mode of the ocean, the tidal response can be much higher than a homogeneous rocky-planet model would predict (see, e.g., Auclair-Desrotour et al. 2019). Investigating this aspect consistently will require us to generalize the tidal formalism used here to account for the frequency dependence of the dynamical tide (e.g., using the formalism of Kaula 1961).

That is why we think that we might need to perform a TTV analysis of the TRAPPIST-1 system accounting for the various physical processes described here with no particular preconception about the values of the parameters for the planetary Love numbers. If the TTVs are reproduced by having a T-1b planet with a high Love number, this could be a sign for a liquid layer on the planet, possibly a magma ocean given the flux it receives and the tidal heat flux it might generate (e.g., Turbet et al. 2018; Makarov et al. 2018).

While a difference of a few $\sim 10 \mathrm{~s}$ is potentially observable, it could be interpreted as a system with slightly different planetary masses and periods by a classical TTV retrieval code. Our group is thus currently investigating if these effects could be picked up with such a retrieval code and, if so, under what conditions (e.g., duration of the observations, precision of the timings). We are also working on implementing these effects in the TTV analysis pipeline and plan to revisit the analysis with the additional parameters mentioned earlier (Grimm et al., in prep.).

Acknowledgements. The authors would like to thank the anonymous referee for helping improving the manuscript. This work has been carried out within the framework of the NCCR PlanetS supported by the Swiss National Science Foundation. B.-O.D. acknowledges support from the Swiss National Science Foundation (PP00P2_163967). P.A.-D. acknowledges financial support from the European Research Council via the Consolidator grant EXOKLEIN (grant number 771620). F.S. acknowledges support from the CNRS/INSU PNP (Programme
National de Planétologie). This research has made use of NASA's Astrophysics Data System.

\section{References}

Agol, E., \& Fabrycky, D. C. 2018, Handbook of Exoplanets (Berlin: Springer), 7 Agol, E., Steffen, J., Sari, R., \& Clarkson, W. 2005, MNRAS, 359, 567

Agol, E., Carey, S., Delrez, L., et al. 2019, Revisiting and refining the model of TRAPPIST-1 with Spitzer (Washington, DC, USA: Spitzer Proposal)

Anderson, J. D., Esposito, P. B., Martin, W., Thornton, C. L., \& Muhleman, D. O. 1975, ApJ, 200, 221

Auclair-Desrotour, P., Leconte, J., Bolmont, E., \& Mathis, S. 2019, A\&A, 629, A132

Blanco-Cuaresma, S., \& Bolmont, E. 2017, EWASS Special Session 4 (2017): Star-planet interactions

Bolmont, E., Raymond, S. N., Leconte, J., Hersant, F., \& Correia, A. C. M. 2015 , A\&A, 583, A116

Bolmont, E., Demory, B.-O., Blanco-Cuaresma, S., et al. 2020, Initial conditions to reproduce figures of the article On the impact of tides on the transit- timing fits to the TRAPPIST- 1 system

Correia, A. C. M., \& Rodríguez, A. 2013, ApJ, 767, 128

Correia, A. C. M., Laskar, J., Farago, F., \& Boué, G. 2011, Celest. Mech. Dyn. Astron., 111, 105

Eggleton, P. P., Kiseleva, L. G., \& Hut, P. 1998, ApJ, 499, 853

Gillon, M., Jehin, E., Lederer, S. M., et al. 2016, Nature, 533, 221

Gillon, M., Triaud, A. H. M. J., Demory, B.-O., et al. 2017, Nature, 542, 456

Grimm, S. L., Demory, B.-O., Gillon, M., et al. 2018, A\&A, 613, A68

Hay, H. C. F. C., \& Matsuyama, I. 2019, ApJ, 875, 22

Heyl, J. S., \& Gladman, B. J. 2007, MNRAS, 377, 1511

Hut, P. 1981, A\&A, 99, 126

Jordán, A., \& Bakos, G. Á. 2008, ApJ, 685, 543

Kaula, W. M. 1961, Geophys. J., 5, 104

Kidder, L. E. 1995, Phys. Rev. D, 52, 821

Luger, R., Sestovic, M., Kruse, E., et al. 2017, Nat. Astron., 1, 0129

Maciejewski, G., Fernández, M., Aceituno, F., et al. 2018, Acta Astron., 68, 371

Makarov, V. V., Berghea, C. T., \& Efroimsky, M. 2018, ApJ, 857, 142

Mignard, F. 1979, Moon Planets, 20, 301

Miralda-Escudé, J. 2002, ApJ, 564, 1019

Murray, C. D., \& Dermott, S. F. 1999, Solar System Dynamics (Cambridge: Cambridge University Press)

Neron de Surgy, O., \& Laskar, J. 1997, A\&A, 318, 975

Newhall, X. X., Standish, E. M., \& Williams, J. G. 1983, A\&A, 125, 150

Newton, E. R., Irwin, J., Charbonneau, D., et al. 2016, ApJ, 821, 93

Pál, A., \& Kocsis, B. 2008, MNRAS, 389, 191

Ragozzine, D., \& Wolf, A. S. 2009, ApJ, 698, 1778

Rein, H., \& Spiegel, D. S. 2015, MNRAS, 446, 1424

Turbet, M., Bolmont, E., Leconte, J., et al. 2018, A\&A, 612, A86

Zahn, J. P. 1975, A\&A, 41, 329 
Appendix A: Initial conditions for the TRAPPIST-1 simulations

Table A.1. Stellar parameters.

\begin{tabular}{ccc}
\hline \hline Parameter & Value & Unit \\
\hline$M_{\star}$ & 0.08 & $M_{\odot}$ \\
$R_{\star}$ & 0.117 & $R_{\odot}$ \\
$r g_{\star}^{2}$ & 0.2 & \\
Rotation period & 3.3 & day \\
\hline
\end{tabular}

To ensure the reproducibility of our simulations, we give here the exact initial conditions we took for the system. Table A.1 gives the stellar parameters used for the integration of the system. The stellar mass and radius come from Gillon et al. (2017) and the rotation comes from Luger et al. (2017). The value of the radius of gyration squared $r g_{\star}^{2}$ (Hut 1981) comes from Bolmont et al. (2015) and should be typical of a fully convective dwarf.

Table A. 2 gives the masses and radii of the planets as well as the initial orbital elements. We consider that all planets have the same radius of gyration squared $r g_{\mathrm{p}}^{2}=0.3308$ (where this quantity is related to the moment of inertia $\left.I_{\mathrm{p}}=M_{\mathrm{p}}\left(R_{\mathrm{p}} r g_{\mathrm{p}}\right)^{2}\right)$. We consider that all planets have a zero obliquity (angle between the direction perpendicular to the orbital plane and the rotation axis of the planet) and that they are tidally locked (see discussion in Luger et al. 2017).

To perform the integration of the system, we used PosidoNIUS v2019.07.30 ${ }^{3}$. This version was slightly altered to be able to fix a maximum time step size (0.01 day). The initial conditions can be found in Bolmont et al. (2020) ${ }^{4}$.

Table A.2. Masses, radii, and initial orbital elements used for the dynamical simulations of the TRAPPIST-1 system.

\begin{tabular}{ccccccccc}
\hline \hline Planet & Mass & Radius & $\begin{array}{c}\text { Semimajor } \\
\text { axis }\end{array}$ & Eccentricity & Inclination & $\begin{array}{c}\text { Mean } \\
\text { anomaly } \\
(\text { degree })\end{array}$ & $\begin{array}{c}\text { Argument of } \\
\text { pericenter } \\
(\text { degree })\end{array}$ & $\begin{array}{c}\text { Longitude of } \\
\text { ascending node } \\
(\text { degree })\end{array}$ \\
\hline b & $2.97733 \mathrm{e}-06$ & 1.127 & 0.01110318 & 0 & 0.59 & 90.0000000 & 0 & 0 \\
c & $3.34950 \mathrm{e}-06$ & 1.100 & 0.01520668 & 0 & 0.50 & 51.5815880 & 0 & 0 \\
$\mathrm{~d}$ & $9.16102 \mathrm{e}-07$ & 0.788 & 0.02142513 & 0 & 0.30 & 84.5759410 & 0 & 0 \\
$\mathrm{e}$ & $2.34751 \mathrm{e}-06$ & 0.915 & 0.02815839 & 0 & 0.40 & 305.455247 & 0 & 0 \\
$\mathrm{f}$ & $2.69105 \mathrm{e}-06$ & 1.052 & 0.03705241 & 0 & 0.08 & 283.559942 & 0 & 0 \\
$\mathrm{~g}$ & $3.29224 \mathrm{e}-06$ & 1.154 & 0.04508048 & 0 & 0.37 & 233.773520 & 0 & 0 \\
$\mathrm{~h}$ & $9.73359 \mathrm{e}-07$ & 0.777 & 0.05955922 & 0 & 0.20 & 1.31390800 & 0 & 0 \\
\hline
\end{tabular}

Notes. The inclination is given here with respect to the equatorial plane of the star. 


\section{Appendix B: Transit timing variations for the four outer planets of TRAPPIST-1}

As in Figs. 1 and B.1 shows the difference in TTVs with the $N$-body case for seven different simulations ( $N$-body and simulations with additional effects) for TRAPPIST-1e to TRAPPIST-1h. General relativity is the dominant effect, but the non-dissipative part of the planetary tidal force (via $k_{2, \mathrm{p}}$ ) and the rotational flattening of the planets (via $k_{2 f, \mathrm{p}}$ ) are still contributing marginally. Only for TRAPPIST- $1 \mathrm{~h}$ is general relativity the only relevant process to account for.
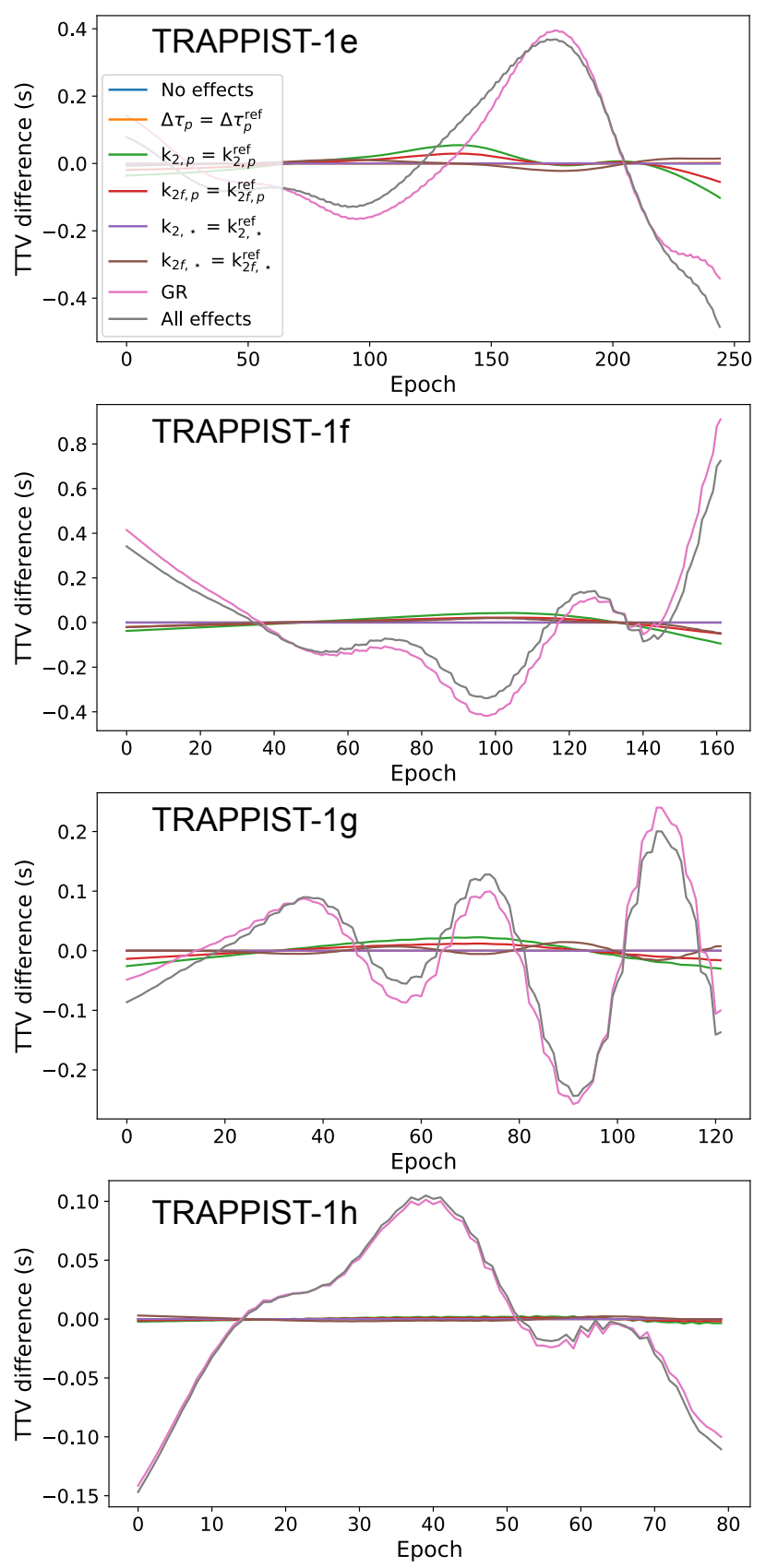

Fig. B.1. As the bottom panels of Fig. 1, but for $(a)$ TRAPPIST-1e, (b) TRAPPIST-1f, (c) TRAPPIST-1g, and ( $d$ ) TRAPPIST-1h. The general relativity is the dominant effect, but the planetary deformation (due to tides or rotation) is not quite completely negligible, except for TRAPPIST-1h. 